\title{
Transient Analysis of Lumped Circuit Networks Loaded Thin Wires By DGTD Method
}

\author{
Ping Li, Student Member, IEEE, , Yifei Shi, Member, IEEE, Li Jun Jiang, Senior Member, IEEE , \\ and Hakan Bağcı, Senior Member, IEEE
}

\begin{abstract}
With the purpose of avoiding very fine mesh cells in the proximity of a thin wire, the modified telegrapher's equations (MTEs) are employed to describe the thin wire voltage and current distributions, which consequently results in reduced number of unknowns and augmented Courant-Friedrichs-Lewy (CFL) number. As hyperbolic systems, both the MTEs and the Maxwell's equations are solved by the discontinuous Galerkin time-domain (DGTD) method. In realistic situations, the thin wires could be either driven or loaded by circuit networks. The thin wire-circuit interface performs as a boundary condition for the thin wire solver, where the thin wire voltage and current used for the incoming flux evaluation involved in the DGTD analyzed MTEs are not available. To obtain this voltage and current, an auxiliary current flowing through the thin wire-circuit interface is introduced at each interface. Corresponding auxiliary equations derived from the invariable property of characteristic variable for hyperbolic systems are developed and solved together with the circuit equations established by the modified nodal analysis (MNA) modality. Furthermore, in order to characterize the field and thin wire interactions, a weighted electric field and a volume current density are added into the MTEs and Maxwell-Ampere's law equation, respectively. To validate the proposed algorithm, three representative examples are presented.
\end{abstract}

Index Terms-Characteristic variable, discontinuous Galerkin time-domain (DGTD) method, hyperbolic system, modified telegrapher's equation (MTE), modified nodal analysis (MNA), thin wire-circuit boundary.

\section{INTRODUCTION}

$\mathbf{F}$ ULL-wave modeling of electromagnetic (EM) systems involving thin wire structures such as cable probes for near-field scanning [1], dipole antennas [2] used for antenna calibration and measurement in the reverberation chamber, etc., requires strong mesh refinements in the peripheral region of the thin wires, consequently resulting in the significan$t$ increase of unknowns and simultaneously very stringent time-step size required by the explicit time-domain marching scheme. To address these problems, various approaches have been proposed in the past years by approximating the thin wire with equivalent models.

One of these approaches is that the thin wire voltage and current are characterized by the modified telegrapher's

P. $\mathrm{Li}$ is with the Department of Electrical and Computer Engineering, Purdue University, West Lafayette, IN 47906, United States.

P. Li, Y. Shi, and H. Bağc1 are with the Division of Computer, Electrical, and Mathematical Sciences and Engineering and the Center for Uncertainty Quantification in Computational Science and Engineering, King Abdullah University of Science and Technology (KAUST), Thuwal, 23955-6900, Saudi Arabia (email: yifei.shi@kaust.edu.sa, hakan.bagci@kaust.edu.sa).

L. J. Jiang is with the Department of Electrical and Electronic Engineering, The University of Hong Kong (HKU), Pokfulam Road, Hong Kong SAR. (email: liping@eee.hku.hk, jianglj@hku.hk) equations (MTEs) [3]-[5]. The MTEs are derived based on the assumption that the EM fields near the thin wire are static and the conservation of charge along the thin wire is satisfied. L. Holland and R. Simpson [3] firstly proposed the MTEs for the thin wire modeling and implemented it into the finite difference time-domain (FDTD) method, where the thin wires are placed along the Cartesian grid. To handle the staircase error, [4] proposed a treatment that is capable of incorporating arbitrarily oriented thin wires into FDTD analysis. Later, the thin wire model was further extended into the finite element method (FEM) [5]. To characterize the interactions between the thin wire and the field solver, a cylindrical coupling zone surrounding the wire is defined. The coupling from the field to the thin wire is achieved by introducing a weighted electric field into the MTEs, and on the other hand a volume current density is added into the Maxwell-Ampere's law equation in order to facilitate the thin wire to field coupling, wherein the radius of the coupling zone is determined by numerical experiments. As another popular method, the material properties (the permittivity and permeability) in the vicinity of the thin wires [6]-[9] are modified to ensure that the EM fields are properly corrected to the original values in the presence of the physical conducting thin wires. Examples of other approaches such as the integral equation based approaches and transmission line method (TLM) based algorithms can be found in [10]-[13].

In this work, the first focus is to model the thin wire based on the MTEs. As a hyperbolic system, the MTEs are numerically solved by the discontinuous Galerkin time-domain (DGTD) method [14], [15]. In the same way, the two firstorder time-derivative Maxwell's equations are analyzed by the DGTD method as well [16], [17]. Like finite volume timedomain method (FVM) [18], [19], DGTD allows solutions to be discontinuous at the interfaces of mesh elements and it uses the numerical flux derived from the Rankine Hugoniot jump relations [19] for the information communication between neighboring elements (The numerical flux in fact can be decomposed into two parts: one is the outgoing flux that only requires solutions in present mesh cell, another is the incoming flux that involves solutions from its neighboring elements). Therefore, all operations of DGTD are spatially local. Furthermore, by approximating the unknowns by hierarchical basis functions, DGTD can achieve same high-order accuracy as FEM [21], [22]. Based on the local operation property, the DGTD resulted mass-matrices are block diagonal, which can be inverted and stored before the time-marching process. Therefore, a compact and efficient solver is readily achieved 


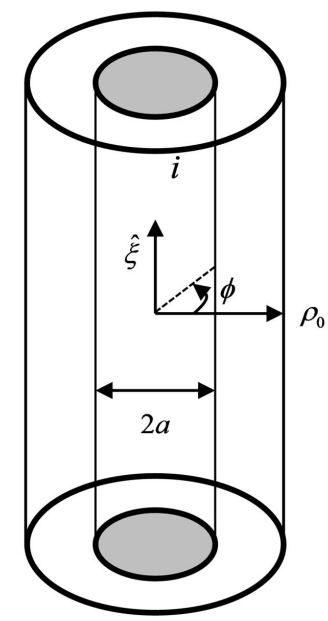

Fig. 1. The schematic illustration of a wire segment $i$ with radius $\rho=a$ oriented in $\hat{\xi}$ direction. To achieve the thin wire and field coupling, a tube region is defined from $\rho=a$ to $\rho_{0}$.

when combined with an explicit time integration scheme.

In many situations, thin wires are interfaced with lumped circuit networks [23]-[26] that might include either passive, active, linear, or nonlinear elements. This interface actually serves as the truncation boundary of the thin wire hyperbolic equations, whereas the thin wire voltage and current used for incoming flux calculation are unfortunately not available at the interface. To address this problem, we introduce an auxiliary current source flowing through this interface. Accordingly, an auxiliary equation is derived based on the fact that the characteristic variables propagate invariably along the characteristic curves for hyperbolic systems [18], [19]. This auxiliary equation will be solved together with the circuit equations constructed by the modified nodal analysis (MNA) method [27]-[29]. Due to the local operation properties of DGTD, the finalized thin wire-circuit coupling matrix is also local, and thus it is very small (The dimension of the wirecircuit matrix is equal to the number of unknowns in the circuit network plus the number of auxiliary current source). As a result, it can be efficiently solved even though nonlinear circuit elements are involved.

In summary, the contribution of this work has two folds. Firstly, the DGTD-Maxwell's equations solver, the thin-wire solver, and the circuit solver are integrated together with the purpose to analyze thin wires terminated by lumped nonlinear circuit networks. Secondly, the treatment of thin wire and circuit interface is elegantly handled based on the invariable property of the characteristic variables.

The remainder of this paper is organized as follows. In Section II, the basic theories and mathematical formulae are given, including the description of the MTEs, the DGTD formulation details, the treatment of thin wire-circuit interface, and the coupling between the MTEs and Maxwell's equations. In section III, numerical examples are presented to validate the proposed algorithm. Conclusions are made at the end of the paper.

\section{THEORY AND MATHEMATICAL FoRMULATION}

In this Section, the MTEs based thin wire model and the enforcement of DG over the MTE are firstly provided. Next, the auxiliary equation for the hybrid thin wire-circuit system is derived. And then, the coupling strategy between the thin wire and Maxwell's equations is given. Finally, the DGTD formulation of the two first-order time-derivative Maxwell's equations is detailed.

\section{A. The MTE Based Thin Wire Model}

Following the Holland-Simpson's thin wire model [3], we assume that the thin wire is a perfectly electrical conductor (PEC) placed along the direction $\hat{\xi}$ and embedded in a homogeneous medium with permittivity $\epsilon$, permeability $\mu$, and conductivity $\sigma$. In the cylindrical coordinate system, the electric field $E_{\rho}$ and magnetic field $H_{\phi}$ are inversely proportional to $\rho$ in the tube region shown in Fig. 1. Namely,

$$
\begin{aligned}
& E_{\rho}(\xi, \rho, t)=C \frac{V(\xi, t)}{2 \pi \epsilon \rho} \\
& H_{\phi}(\xi, \rho, t)=\frac{I(\xi, t)}{2 \pi \rho}
\end{aligned}
$$

with $C, V$, and $I$ denoting the per-unit-length (p.u.l) capacitance, thin wire voltage, and current, respectively.

By substituting (1) and (2) into the $\phi$-component of the Maxwell-Faraday's law equation and acknowledging the conservation of the charge along the thin wire, we can obtain the two MTEs after a few mathematical operations [3], [4]:

$$
\begin{aligned}
& C \frac{\partial V}{\partial t}+\frac{\partial I}{\partial \xi}+G V=0 \\
& L \frac{\partial I}{\partial t}+\frac{\partial V}{\partial \xi}=0
\end{aligned}
$$

where the impressed voltage and current sources along the thin wire are not considered for simplicity but without losing of generality. $L=\frac{\mu}{2 \pi} \log \left(\frac{\rho_{0}+a}{2 a}\right)$ and $G=C \sigma / \epsilon$ are the p.u.l inductance and conductance, respectively. The current propagates along the thin wire with speed $c=\frac{1}{\sqrt{L C}}$.

In order to apply the DGTD over the MTEs, we first split the thin wire into non-overlapping segments $T_{i}\left(i=1, \cdots, N_{\mathrm{w}}\right)$ bound by $\partial_{T_{i}}$. In segment $i$, we write (3) and (4) as a conservation form [18], [19]:

$$
\frac{\partial \mathbf{U}_{i}}{\partial t}+\nabla \cdot \mathcal{F}\left(\mathbf{U}_{i}\right)+\overline{\overline{\mathcal{G}}}_{i} \cdot \mathbf{U}_{i}=0,
$$

where $\mathbf{U}_{i}=\left[V^{i}, I^{i}\right]^{T}, \nabla \cdot=\frac{\partial}{\partial \xi}$ for 1-D problem, $\mathcal{F}\left(\mathbf{U}_{i}\right)=$ $\overline{\overline{\mathcal{A}}}_{i} \cdot \mathbf{U}_{i}$ with

$$
\overline{\overline{\mathcal{A}}}_{i}=\left[\begin{array}{cc}
0 & 1 / C^{i} \\
1 / L^{i} & 0
\end{array}\right]
$$

and

$$
\overline{\overline{\mathcal{G}}}_{i}=\left[\begin{array}{cc}
G^{i} / C^{i} & 0 \\
0 & 0
\end{array}\right] .
$$

The process of finding the unique solution of (5) with the piecewise constant initial value is termed as the Riemann problem. To obtain the unique solution, the numerical flux 


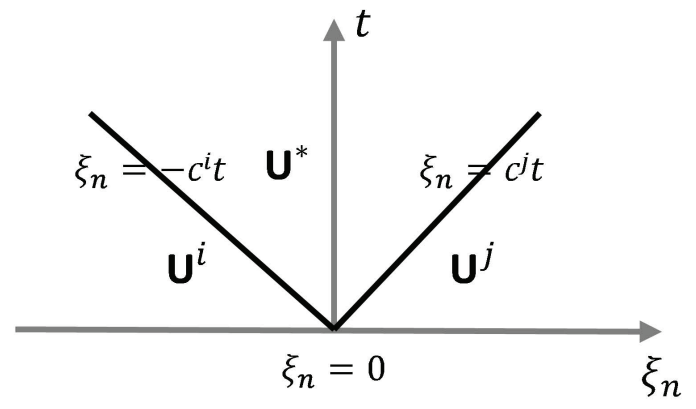

Fig. 2. The characteristic plot of the 1-D thin wire system. The horizontal axis $\xi_{n}$ means the displacement along the normal direction at the interface of neighboring segments, and the vertical axis represents the time variable. $\mathbf{U}^{i}=\left[\begin{array}{ll}V^{i} & I^{i}\end{array}\right]^{T}$ and $\mathbf{U}^{j}=\left[\begin{array}{ll}V^{j} & I^{j}\end{array}\right]^{T}$ are two initial solutions in the present and its neighboring elements. Due to the discontinuity of solutions at the interface of neighboring elements, an intermediate variable $\mathbf{U}^{*}=\left[V^{*} I^{*}\right]^{T}$ that is a function of $\mathbf{U}^{i}$ and $\mathbf{U}^{j}$ is introduced. To have a unique solution, the explicit expression of $\mathbf{U}^{*}$ is determined by the Rankine-Hugoniot jump relations.

derived according to the Rankine Hugoniot jump relation$s$ [18], [19] is introduced to facilitate the information exchange between neighboring elements. It starts with finding out the characteristics of the hyperbolic system that are determined by the eigenvalues of the flux matrix $\breve{\mathcal{A}}_{i}$ defined as the product between the unit outward normal vector $k_{\xi}^{i}\left(k_{\xi}^{i}=1\right.$ or -1 for 1-D problem) and $L C$ dependent matrix $\overline{\overline{\mathcal{A}}}_{i}$.

For the hyperbolic partial differential equations (PDE), the derivation of the numerical flux is started from the decomposition of the flux matrix $\breve{\mathcal{A}}$ into the product of the eigenvectors and eigenvalues. Namely,

$$
\breve{\mathcal{A}}=\overline{\overline{\mathcal{R}}}_{i} \overline{\bar{\Lambda}}_{i} \overline{\overline{\mathcal{R}}}_{i}^{-1},
$$

where $\overline{\mathcal{R}}_{i}$ and $\overline{\mathcal{R}}_{i}^{-1}$ are the right and the left eigenvectors of the flux matrix $\breve{\mathcal{A}}_{i}$, respectively; $\overline{\bar{\Lambda}}_{i}$ is a diagonal matrix containing real eigenvalues that equal to the gradient of the characteristic curves involved in the derivation of numerical flux. They are defined as:

$$
\begin{aligned}
& \overline{\overline{\mathcal{R}}}_{i}=\left[\begin{array}{cc}
1 & 1 \\
-k_{\xi}^{i} \tilde{Y}^{i} & k_{\xi}^{i} \tilde{Y}^{i}
\end{array}\right], \\
& \overline{\overline{\mathcal{R}}}_{i}^{-1}=\frac{1}{2}\left[\begin{array}{cc}
1 & -k_{\xi}^{i} \tilde{Z}^{i} \\
1 & k_{\xi}^{i} \tilde{Z}^{i}
\end{array}\right],
\end{aligned}
$$

and

$$
\overline{\bar{\Lambda}}_{i}=\left[\begin{array}{cc}
-c^{i} & 0 \\
0 & c^{i}
\end{array}\right]
$$

with $\tilde{Z}^{i}=\sqrt{L^{i} / C^{i}}$ and $\tilde{Y}^{i}=\sqrt{C^{i} / L^{i}}$ denoting the characteristic impedance and admittance, respectively. To obtain the expression of the numerical flux, the following jump relations across the characteristics (as shown in Fig. 2) must be satisfied [19]:

1) Jump across the characteristic curve $\xi_{n}=-c^{i} t$

$$
\begin{aligned}
& \frac{k_{\xi}^{i}}{C^{i}}\left(I^{*}-I^{i}\right)=-c^{i}\left(V^{*}-V^{i}\right) \\
& \frac{k_{\xi}^{i}}{L^{i}}\left(V^{*}-V^{i}\right)=-c^{i}\left(I^{*}-I^{i}\right)
\end{aligned}
$$

2) Jump across the characteristic curve $\xi_{n}=c^{j} t$

$$
\begin{aligned}
& \frac{k_{\xi}^{i}}{C^{j}}\left(I^{*}-I^{j}\right)=c^{j}\left(V^{*}-V^{j}\right) \\
& \frac{k_{\xi}^{i}}{L^{j}}\left(V^{*}-V^{j}\right)=c^{j}\left(I^{*}-I^{j}\right)
\end{aligned}
$$

where the superscript $j$ denotes the neighboring element of the present element $i, c^{i}$ and $c^{j}$ represent the characteristic speeds in the current element $i$ and its neighbor $j$, respectively; $V^{*}$ and $I^{*}$ are two intermediate states (they are functions of parameters: $V^{i}, V^{j}, I^{i}$, and $I^{j}$.).

Adding $-k_{\xi}^{i} \cdot(12)$ and $k_{\xi}^{i} \cdot(14)$ and rearranging the terms yields the final expression for the PDE in (4):

$$
k_{\xi}^{i} \cdot V^{*}=\frac{\left(I^{i}+k_{\xi}^{i} \tilde{Y}^{i} V^{i}\right)-\left(I^{j}-K_{\xi}^{i} \tilde{Y}^{j} V^{j}\right)}{\tilde{Y}^{i}+\tilde{Y}^{j}}
$$

Following the same procedure, we can get the flux expression for the PDE in (3) by adding $-k_{\xi}^{i} \cdot(13)$ and $k_{\xi}^{i} \cdot(15)$ :

$$
k_{\xi}^{i} \cdot I^{*}=\frac{\left(V^{i}+k_{\xi}^{i} \tilde{Z}^{i} I^{i}\right)-\left(V^{j}-k_{\xi}^{i} \tilde{Z}^{j} I^{j}\right)}{\tilde{Z}^{i}+\tilde{Z}^{j}}
$$

To obtain the DGTD formulated matrix equations, we firstly apply the DG testing over (3) and (4) in element $i$ and further resort to the integration by parts and the Gaussian theorem, we can achieve

$$
\begin{gathered}
\int_{T_{i}} \varphi_{p}^{i}(\xi) \cdot\left(C^{i} \frac{\partial V^{i}}{\partial t}+\frac{\partial I^{i}}{\partial \xi}+G^{i} V^{i}\right) d \xi= \\
\int_{\partial T_{i}} \varphi_{p}^{i}(\xi) \cdot\left[k_{\xi}^{i} \cdot\left(I^{i}-I^{*}\right)\right] d \xi \\
\int_{T_{i}} \psi_{q}^{i}(\xi) \cdot\left(L^{i} \frac{\partial I^{i}}{\partial t}+\frac{\partial V^{i}}{\partial \xi}\right) d \xi= \\
\int_{\partial T_{i}} \psi_{q}^{i}(\xi) \cdot\left[k_{\xi}^{i} \cdot\left(V^{i}-V^{*}\right)\right] d \xi
\end{gathered}
$$

where $\varphi_{p}^{i}(\xi)$ and $\psi_{q}^{i}(\xi)$ denote the $p$ - and $q$-th 1-D testing functions for $V^{i}$ and $I^{i}$, respectively. The introduction of $I^{*}$ and $U^{*}$ is due to the fact that the solutions at the interface of neighboring elements are allowed to be discontinuous in the DGTD analysis, and the values of them are determined by the above discussed Rankine-Hugoniot jump relations (see eqns. from (12) to (17)). Compared with the continuous Galerkin testing, it is interesting to note that the integral operations involved in the right hand sides of (18) and (19) for DG testing are implemented at the boundary face of each element instead of the global boundary.

Next, by approximating the thin wire voltage and current with 1-D basis functions as [21]: $V^{i}=\sum_{p=1}^{n_{i}^{V}} f_{p}^{i}(t) \varphi_{p}^{i}(\xi)$ and $I^{i}=\sum_{q=1}^{n_{i}^{I}} g_{q}^{i}(t) \psi_{q}^{i}(\xi)$, and then substitute them together with (16) and (17) into (18) and (19), we can obtain the semidiscrete matrix equations as

$$
\begin{aligned}
& \overline{\mathbf{M}}_{V}^{i} \frac{\partial \bar{f}^{i}}{\partial t}+\overline{\mathbf{S}}_{I}^{i} \bar{g}^{i}+\overline{\mathbf{G}}_{V}^{i} \bar{f}^{i}= \\
& \overline{\mathbf{F}}_{V I}^{i i} \bar{g}^{i}+\overline{\mathbf{F}}_{V V}^{i i} \bar{f}^{i}+\overline{\mathbf{F}}_{V I}^{i j} \bar{g}^{j}+\overline{\mathbf{F}}_{V V}^{i j} \bar{f}^{j}
\end{aligned}
$$




$$
\begin{aligned}
& \overline{\mathbf{M}}_{I}^{i} \frac{\partial \bar{g}^{i}}{\partial t}+\overline{\mathbf{S}}_{V}^{i} \bar{f}^{i}= \\
& \\
& \overline{\mathbf{F}}_{I V}^{i i} \bar{f}^{i}+\overline{\mathbf{F}}_{I I}^{i i} \bar{g}^{i}+\overline{\mathbf{F}}_{I V}^{i j} \bar{f}^{j}+\overline{\mathbf{F}}_{I I}^{i j} \bar{g}^{j}
\end{aligned}
$$

where $\bar{f}^{i / j}$ and $\bar{g}^{i / j}$ are two column vectors containing the time-dependent coefficients of basis functions, and the elements in the above matrices are defined by

$$
\begin{aligned}
& {\left[\overline{\mathbf{M}}_{V}^{i}\right]_{k l}=C^{i} \int_{T_{i}} \varphi_{k}^{i} \cdot \varphi_{l}^{i} d \xi} \\
& {\left[\overline{\mathbf{M}}_{I}^{i}\right]_{k l}=L^{i} \int_{T_{i}} \psi_{k}^{i} \cdot \psi_{l}^{i} d \xi} \\
& {\left[\overline{\mathbf{S}}_{I}^{i}\right]_{k l}=\int_{T_{i}} \varphi_{k}^{i} \cdot \frac{\partial \psi_{l}^{i}}{\partial \xi} d \xi} \\
& {\left[\overline{\mathbf{S}}_{V}^{i}\right]_{k l}=\int_{T_{i}} \psi_{k}^{i} \cdot \frac{\partial \varphi_{l}^{i}}{\partial \xi} d \xi} \\
& {\left[\overline{\mathbf{G}}_{V}^{i}\right]_{k l}=G^{i} \int_{T_{i}} \varphi_{k}^{i} \cdot \varphi_{l}^{i} d \xi} \\
& {\left[\overline{\mathbf{F}}_{V I}^{i i}\right]_{k l}=\frac{k_{\xi}^{i} \tilde{Z}^{j}}{\tilde{Z}^{i}+\tilde{Z}^{j}} \int_{\partial T_{i}} \varphi_{k}^{i} \cdot \psi_{l}^{i} d \xi} \\
& {\left[\overline{\mathbf{F}}_{V V}^{i i}\right]_{k l}=\frac{-1}{\tilde{Z}^{i}+\tilde{Z}^{j}} \int_{\partial T_{i}} \varphi_{k}^{i} \cdot \varphi_{l}^{i} d \xi} \\
& {\left[\overline{\mathbf{F}}_{V I}^{i j}\right]_{k l}=\frac{-k_{\xi}^{i} \tilde{Z}^{j}}{\tilde{Z}^{i}+\tilde{Z}^{j}} \int_{\partial T_{i}} \varphi_{k}^{i} \cdot \psi_{l}^{j} d \xi} \\
& {\left[\overline{\mathbf{F}}_{V V}^{i j}\right]_{k l}=\frac{1}{\tilde{Z}^{i}+\tilde{Z}^{j}} \int_{\partial T_{i}} \varphi_{k}^{i} \cdot \varphi_{l}^{j} d \xi} \\
& {\left[\overline{\mathbf{F}}_{I V}^{i i}\right]_{k l}=\frac{k_{\xi}^{i} \tilde{Y}^{j}}{\tilde{Y}^{i}+\tilde{Y}^{j}} \int_{\partial T_{i}} \psi_{k}^{i} \cdot \varphi_{l}^{i} d \xi} \\
& {\left[\overline{\mathbf{F}}_{I I}^{i i}\right]_{k l}=\frac{-1}{\tilde{Y}^{i}+\tilde{Y}^{j}} \int_{\partial T_{i}} \psi_{k}^{i} \cdot \psi_{l}^{i} d \xi} \\
& {\left[\overline{\mathbf{F}}_{I V}^{i j}\right]_{k l}=\frac{-k_{\xi}^{i} \tilde{Y}^{j}}{\tilde{Y}^{i}+\tilde{Y}^{j}} \int_{\partial T_{i}} \psi_{k}^{i} \cdot \varphi_{l}^{j} d \xi} \\
& {\left[\overline{\mathbf{F}}_{I I}^{i j}\right]_{k l}=\frac{1}{\tilde{Y}^{i}+\tilde{Y}^{j}} \int_{\partial T_{i}} \psi_{k}^{i} \cdot \psi_{l}^{j} d \xi}
\end{aligned}
$$

To solve the above matrix equations in (20) and (21), the fourth-order Runge-Kutta (RK) method is applied. The stability condition is determined by

$$
\delta t_{w} \leq \min \left\{h_{\text {min }} \sqrt{L^{i} C^{i}} / 4(\tilde{p}+1)^{2}\right\}
$$

where $\tilde{p}$ is the order of basis functions, and $h_{\min }$ denotes the minimum length of wire segments.

Since the incorporation of boundary conditions into DGTD analysis is achieved by revising the numerical flux, the flux expressions in (16) and (17) corresponding to the short- and open-circuit cases must be carefully addressed. Suppose the element $i$ has no right neighbor, for open-circuit case, the current from the neighboring element $j$ is zero $\left(I^{j}=0\right)$ and thereby the characteristic impedance $Z^{j}$ can be regarded as infinity. As a result, the numerical fluxes in (16) and (17) for open-circuit situation are revised to be

$$
\begin{aligned}
& k_{\xi}^{i} \cdot V^{*}=k_{\xi}^{i} V^{i}+\frac{I^{i}}{\tilde{Y}^{i}} \\
& k_{\xi}^{i} \cdot I^{*}=0
\end{aligned}
$$

The two expressions above can be equivalently achieved by letting $I^{j}=-I^{i}, V^{j}=V^{i}$, and $\tilde{Y}^{j}=\tilde{Y}^{i}$, which makes programming more convenient and easy.

For the short-circuit situation, the voltage from the neighboring element $j$ is zero and the characteristic admittance can be regarded as infinity. In a similar way, the numerical fluxes in (16) and (17) are modified as

$$
\begin{aligned}
& k_{\xi}^{i} \cdot V^{*}=0 \\
& k_{\xi}^{i} \cdot I^{*}=k_{\xi}^{i} I^{i}+\frac{V^{i}}{\tilde{Z}^{i}}
\end{aligned}
$$

To achieve the above two expressions, we can equivalently set $I^{j}=I^{i}, V^{j}=-V^{i}$, and $\tilde{Z}^{j}=\tilde{Z}^{i}$.

As for the thin wire-circuit interface case, $V^{j}$ and $I^{j}$ required for the incoming flux computation flowing from the neighboring element $j$ are acquired from the MNA based circuit equations. Details will be presented in the next part.

\section{B. Treatment of Thin Wire-Circuit Interface}

With regarding to the situation that thin wires are interfaced with circuit networks, the voltage $V^{j}$ and the current $I^{j}$ are not available to evaluate the incoming flux in (16) and (17). To obtain these two values, auxiliary current sources at wirecircuit interfaces are introduced. Firstly, to model the circuit networks, we apply the Kirchoff's current law based MNA method to every non-reference voltage node [28], [29]. The resultant circuit matrix equation at time $t=(n+1) \Delta t$ is

$$
\left[\begin{array}{cc}
{[\mathbf{G}]} & {[\mathbf{B}]} \\
{[\mathbf{B}]^{\mathrm{T}}} & {[\mathbf{D}]}
\end{array}\right]\left[\begin{array}{c}
\mathbf{V}_{n+1}^{\mathrm{CKT}} \\
\mathbf{I}_{n+1}^{\mathrm{CKT}}
\end{array}\right]+\mathbf{I}_{n+1}^{\mathrm{CKT}, \mathrm{nl}}\left(\mathbf{V}_{n+1}^{\mathrm{CKT}}\right)=\left[\begin{array}{c}
\mathbf{I}_{n}^{\mathrm{CP}}+\mathbf{I}_{n+1}^{\mathrm{ind}} \\
\mathbf{V}_{n+1}^{\mathrm{ind}}
\end{array}\right],
$$

where the admittance matrix $[\mathbf{G}]$ is determined by interconnections between circuit elements, $[\mathbf{B}]$ is determined by the connection of supplied voltage sources with only 0,1 and 1 elements, $[\mathbf{D}]$ is equal to zero if there are no controlled sources. $\mathbf{V}_{n+1}^{\text {CKT }}$ denotes the unknown node voltages. $\mathbf{I}_{n+1}^{\text {CKT }}$ denotes the unknown currents through voltage sources. $\mathbf{I}_{n+1}^{\mathrm{CKT}, n l}$ represents currents through branches containing non-linear elements. $\mathbf{I}_{n}^{\mathrm{CP}}$ is comprised of current sources at $t=n \Delta t$ derived from companion models of inductors and capacitors based on the trapezoidal integration rule. $\mathbf{I}_{n+1}^{\text {ind }}$ denotes the independent current sources like the Norton current source. $\mathbf{V}_{n+1}^{\text {ind }}$ represents independent voltage sources in the circuit subsystem like the Thevenin voltage source. The overall dimension of the circuit subsystem in (27), denoted as $N^{\mathrm{CKT}}$, is equal to the number of voltage nodes plus the number of voltage sources.

Due to the introduction of auxiliary current sources, auxiliary equations must be provided accordingly. In this work, they are derived from the fact that the characteristic variables for hyperbolic systems are invariable along the characteristics. For the thin wire hyperbolic system, the characteristic variables for element $i$ are given by [18], [20]

$$
\mathcal{W}_{i, k_{\xi}^{i}}^{\mp}=\overline{\overline{\mathcal{R}}}_{i}^{-1} \mathbf{U}_{i}=\left(V^{i} \mp k_{\xi}^{i} \tilde{Z}^{i} I^{i}\right) / 2
$$

where $\mathcal{W}_{i, k_{\xi}^{i}}^{-}$and $\mathcal{W}_{i, k_{\xi}^{i}}^{+}$represent the incoming and outgoing characteristic variables, respectively. 


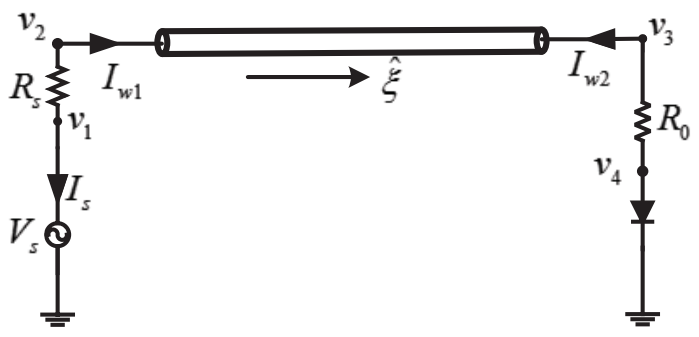

Fig. 3. Schematic Illustration of the thin wire-circuit interface driven by a voltage source with a source resistor $R_{s}$ and loaded by a nonlinear circuit network containing a resistor and a diode. At the thin wire-circuit interface, two auxiliary currents $I_{w 1}$ and $I_{w 2}$ flowing into the thin wire are introduced.

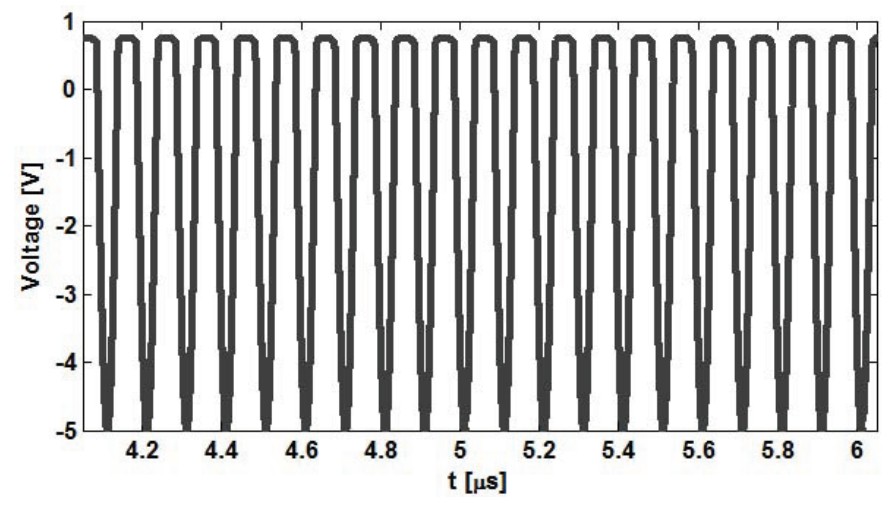

(a)

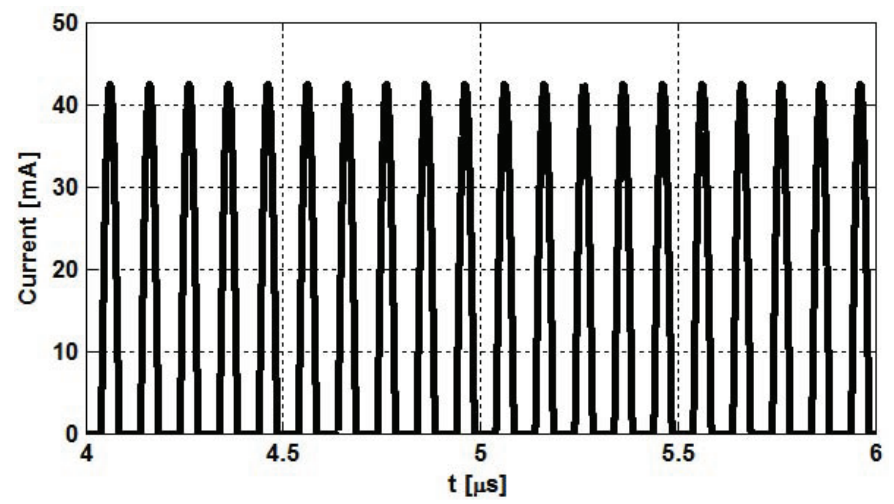

(b)

Fig. 4. The calculated voltage (a) and current (b) across the diode by the proposed thin wire-circuit solver.

Based on the continuous property of the characteristic variables [18], [20], we have the relations

$$
\begin{aligned}
& \mathcal{W}_{j, k_{\xi}^{j}}^{-}=\mathcal{W}_{i, k_{\xi}^{i}}^{+} \\
& \mathcal{W}_{j, k_{\xi}^{j}}^{+}=\mathcal{W}_{i, k_{\xi}^{i}}^{-}
\end{aligned}
$$

with $k_{\xi}^{j}=-k_{\xi}^{i}$. The physical meaning of the above equation is that the outgoing/incoming characteristics in the element $i$ is equivalent to the incoming/outgoing characteristics in its neighboring element $j$.

With above theories, to achieve the coupling between the thin wire and circuit networks, we always use the equation for the incoming characteristic variable $\mathcal{W}_{j,-k_{\xi}^{i}}^{-}$as the auxiliary equation. To have a better insight into the coupling process, a representative example is shown in Fig. 3. It is supposed that the left terminal of the first segment of the thin wire is driven by a voltage source, while the right terminal of the last segment of the thin wire is loaded by a nonlinear circuit network composed of a resistor and a diode with $I_{d}=I_{0}\left[\exp \left(v_{4} / V_{0}\right)-1\right]$. Thus, the finalized circuit matrix equation at the left wire-circuit interface $\left(k_{\xi}^{1}=-1\right)$ is

$$
\left[\begin{array}{cccc}
\frac{1}{R_{\mathrm{s}}} & -\frac{1}{R_{s}} & 0 & 1 \\
-\frac{1}{R_{s}} & \frac{1}{R_{s}} & 1 & 0 \\
1 & 0 & 0 & 0 \\
0 & 1 & -\tilde{Z}^{1} & 0
\end{array}\right]\left[\begin{array}{c}
v_{1} \\
v_{2} \\
I_{w 1} \\
I_{s}
\end{array}\right]=\left[\begin{array}{c}
0 \\
0 \\
V_{s} \\
2 \mathcal{W}_{1,-1}^{+}
\end{array}\right]
$$

, and the finalized circuit matrix at the right wire-circuit interface $\left(k_{\xi}^{N_{\mathrm{w}}}=1\right)$ is

$$
\left[\begin{array}{ccc}
\frac{1}{R_{9}} & -\frac{1}{R_{0}} & 1 \\
-\frac{1}{R_{0}} & \frac{1}{R_{0}} & 0 \\
1 & 0 & -\tilde{Z}^{N_{\mathrm{w}}}
\end{array}\right]\left[\begin{array}{c}
v_{3} \\
v_{4} \\
I_{w 2}
\end{array}\right]+\left[\begin{array}{c}
0 \\
I_{d} \\
0
\end{array}\right]=\left[\begin{array}{c}
0 \\
0 \\
2 \mathcal{W}_{N_{\mathrm{w}}, 1}^{+}
\end{array}\right]
$$

In (32), the minus sign in front of $\tilde{Z}_{N_{\mathrm{w}}}$ is due to the opposite reference directions between the thin wire and the auxiliary current $I_{w 2}$.

To validate the thin wire-circuit solver, the hybrid thin wirecircuit structure in Fig. 3 are investigated. The thin wire is 41 $\mathrm{m}$ and has been split into 164 segments, and the quadratic interpolation functions are used in this work to expand $V$ and $I\left(n_{i}^{V}=n_{i}^{I}=3\right)$. The PUL inductance $L^{i}$ and capacitance $C^{i}$ are given as $L^{i}=0.1 \mathrm{nH}$ and $C^{i}=1.0 \mathrm{nF}$, the source and load resistors are both equal to $50 \Omega$. The thin wire is driven by a sinusoidal voltage source defined as $V_{s}=5.0 \sin \left[2 \pi f_{0}(t-t 0)\right]$ with $f_{0}=10 \mathrm{MHz}$. The voltage drop and the current across the diode are evaluated and plotted in Fig. 4. As it can be seen, the forward voltage drop is limited around $0.7 \mathrm{~V}$, which is consistent with the physical principle. Due to the nonlinear property of diode, we use the Newton-Raphson method to iteratively solve the matrix equation in (32).

\section{Thin Wire and Field Coupling}

Based on the F. Edelvik's model [4], the coupling from the field to the thin wire system is achieved by placing a weighted electric field $\left\langle E_{\xi}^{i}\right\rangle=\int_{0}^{2 \pi} \int_{0}^{\rho_{0}} E_{\xi}(\rho, \phi) \mathfrak{g}(\rho) \rho d \phi d \rho$ (serving as a voltage source) in the tube region surrounding the wire segment $i$ into the right hand side of (4), where $\mathfrak{g}(\rho)$ is a weighting function which is zero for $\rho<a$ and $\rho>\rho_{0}$ [4], [5]. After facilitating the DG testing, we have $\int_{T_{i}} \psi_{k}^{i} \cdot\left\langle E_{\xi}^{i}\right\rangle d \xi=\int_{\Omega_{j}} \psi_{k}^{i} \cdot\left[\mathbf{E}(\mathbf{r}) \cdot \hat{\xi}^{i}\right] \mathfrak{g}(\rho) d V$, where $\Omega_{j}$ is the volume mesh cell generated by solving 3-D Maxwell's equations (In this work, tetrahedrons are used), and $j$ is the global index of the tetrahedrons. Since there will be multiple tetrahedrons in the coupling zone of the wire segment $i$, thus the dimension of the established field to thin wire coupling matrix $\overline{\mathbf{C}}_{\mathrm{EW}}^{i}$ is $n_{i}^{I}$ by $n_{e}^{j} \times N_{c}^{i}$, where $n_{e}^{j}$ is the number of edge vector basis functions $\boldsymbol{\Phi}(\mathbf{r})$ used for the electric field $\mathbf{E}$ expansion in the $j$-th tetrahedron (We approximate both $\mathbf{E}$ and $\mathbf{H}$ in each tetrahedron by twelve hierarchical edge vector basis functions i.e. $n_{e}^{j}=n_{h}^{j}=12$ [31], [32], specifically the first six vector basis functions are tangentially constant but normally 
linear while both the tangential and the normal components of the additional six vector basis functions are varying linearly.), $N_{c}^{i}$ is the total number of tetrahedrons included in the coupling region of the $i$-th wire segment. By rearranging the global index $j$ of the tetrahedrons to be $j^{\prime}=1, \cdots, N_{c}^{i}$, the $k l$ th element of the field to thin wire coupling matrix can be obtained as

$$
\left[\overline{\mathbf{C}}_{\mathrm{EW}}^{i}\right]_{k l}=\int_{\Omega_{j^{\prime}}} \psi_{k}^{i}(\xi) \cdot\left[\boldsymbol{\Phi}_{q}^{j^{\prime}}(\mathbf{r}) \cdot \hat{\xi}^{i}\right] \mathfrak{g}(\rho) d \mathbf{r}
$$

where $l=12\left(j^{\prime}-1\right)+q$ with $q=1, \cdots, 12$.

On the other hand, to achieve the coupling from the thin wire to field system, a weighted volume current density (serving as a radiation source) defined as

$$
\mathbf{J}_{\xi}=I^{j}(t, \xi) \mathfrak{g}(\rho) \hat{\xi}^{j}=\sum_{q=1}^{n_{j}^{I}} g_{q}^{j}(t) \psi_{q}^{j}(\xi) \mathfrak{g}(\rho) \hat{\xi}^{j}
$$

is added into the Maxwell-Ampere's law equation. Similarly, for each tetrahedron in the coupling region, there would be multiple wire segments involved. We denote the number of wire segments coupled to the tetrahedron $i$ is $M_{c}^{i}$. By rearranging the global index $j$ of the wire segments to be $j^{\prime}=1, \cdots, M_{c}^{i}$, and with DG testing over the MaxwellAmpere's law equation, we can obtain the expression of the $k l$-th element of the thin wire to field coupling matrix as

$$
\left[\overline{\mathbf{C}}_{\mathrm{WE}}^{i}\right]_{k l}=\int_{\Omega_{i}}\left[\boldsymbol{\Phi}_{k}^{i}(\mathbf{r}) \cdot \hat{\xi}^{j^{\prime}}\right] \cdot \psi_{q}^{j^{\prime}} \mathfrak{g}(\rho) d \mathbf{r}
$$

where $l=3\left(j^{\prime}-1\right)+q$ with $q=1, \cdots, 3$. Thus, the dimension of this matrix is $n_{e}^{i} \times\left(\sum_{j^{\prime}=1}^{M_{c}} n_{j^{\prime}}^{I}\right)=12 \times\left(3 M_{c}^{i}\right)$.

\section{DGTD Formulation of Maxwell's Equations}

To apply for the DGTD over Maxwell's equations, we first split the computational domain of interest $\Omega$ bound by $\partial \Omega$ into $N_{3 D}$ non-overlapping tetrahedrons $\Omega_{i}$ with boundary face $\partial \Omega_{i}$. In each tetrahedron, the EM fields $\mathbf{E}$ and $\mathbf{H}$ are expanded by edge vector basis functions: $\mathbf{E}^{i}(\mathbf{r}, t)=\sum_{k=1}^{n_{e}^{i}} e_{k}^{i}(t) \Phi_{k}^{i}(\mathbf{r})$ and $\mathbf{H}^{i}(\mathbf{r}, t)=\sum_{l=1}^{n_{h}^{i}} h_{l}^{i}(t) \Psi_{l}^{i}(\mathbf{r})$. By conducting the DG testing over the two first-order time-derivative Maxwell's equations in element $i$ and with the integration by parts and the Gaussian theorem, we can obtain [15], [16], [35]

$$
\begin{aligned}
\int_{\Omega_{i}} \boldsymbol{\Phi}_{k}^{i} \cdot\left[\epsilon_{i} \frac{\partial \mathbf{E}^{i}}{\partial t}-\nabla \times \mathbf{H}^{i}-\mathbf{J}_{\xi}\right] d V= \\
\sum_{f=1}^{4} \int_{\partial \Omega_{i, f}} \boldsymbol{\Phi}_{k}^{i} \cdot\left[\hat{\mathbf{n}}_{i, f} \times\left(\mathbf{H}_{f}^{*}-\mathbf{H}^{i}\right)\right] d S \\
\int_{\Omega_{i}} \mathbf{\Psi}_{l}^{i} \cdot\left[\mu_{i} \frac{\partial \mathbf{H}^{i}}{\partial t}+\nabla \times \mathbf{E}_{i}\right] d V= \\
\sum_{f=1}^{4} \int_{\partial \Omega_{i, f}} \mathbf{\Psi}_{l}^{i} \cdot\left[\hat{\mathbf{n}}_{i, f} \times\left(\mathbf{E}^{i}-\mathbf{E}_{f}^{*}\right)\right] d S
\end{aligned}
$$

where $\mathbf{J}_{\xi}$ the volume current density [see (34)] coupled from the thin wire solver, which is equal to zero if the present tetrahedron $i$ is not falling into the coupling zone. $\hat{\mathbf{n}} \times \mathbf{H}^{*}$ and $\hat{\mathbf{n}} \times \mathbf{E}^{*}$ are termed as numerical flux. According to the Rankine Hugoniot jump relations, we have

$\hat{\mathbf{n}}_{i, f} \times \mathbf{H}_{f}^{*}=\hat{\mathbf{n}}_{i, f} \times\left[\frac{\left(Z^{i} \mathbf{H}^{i}+Z_{f}^{j} \mathbf{H}_{f}^{j}\right)+\hat{\mathbf{n}}_{i, f} \times\left(\mathbf{E}^{i}-\mathbf{E}_{f}^{j}\right)}{Z^{i}+Z_{f}^{j}}\right]$

$\hat{\mathbf{n}}_{i, f} \times \mathbf{E}_{f}^{*}=\hat{\mathbf{n}}_{i, f} \times\left[\frac{\left(Y^{i} \mathbf{E}^{i}+Y_{f}^{j} \mathbf{E}_{f}^{j}\right)+\hat{\mathbf{n}}_{i, f} \times\left(\mathbf{H}_{f}^{j}-\mathbf{H}^{i}\right)}{Y^{i}+Y_{f}^{j}}\right]$

with $\hat{\mathbf{n}}_{i, f}$ denoting the outward unit normal vector at $f$-th face of element $i, Z^{i / j}=\sqrt{\mu^{i / j} / \epsilon^{i / j}}$ and $Y^{i / j}=1 / Z^{i / j}$ denoting the characteristic impedance and admittance, respectively.

Substituting (38), and (39) into (36) and (37), two semidiscrete time-domain matrix equations can be obtained:

$$
\begin{aligned}
& \overline{\mathbf{M}}_{\mathrm{e}}^{i} \frac{\partial \mathbf{e}^{i}}{\partial t}= \overline{\mathbf{S}}_{\mathrm{e}}^{i} \mathbf{h}^{i}+\overline{\mathbf{C}}_{\mathrm{WE}}^{i} \bar{g}^{j^{\prime}}+ \\
& \sum_{f=1}^{4}\left(\overline{\mathbf{F}}_{e e}^{i i, f} \mathbf{e}_{f}^{i}+\overline{\mathbf{F}}_{e e}^{i j, f} \mathbf{e}_{f}^{j}+\overline{\mathbf{F}}_{e h}^{i i, f} \mathbf{h}_{f}^{i}+\overline{\mathbf{F}}_{e h}^{i j, f} \mathbf{h}_{f}^{j}\right) \\
& \overline{\mathbf{M}}_{\mathrm{h}}^{i} \frac{\partial \mathbf{h}^{i}}{\partial t}=-\overline{\mathbf{S}}_{\mathrm{h}}^{i} \mathbf{e}^{i}+ \\
& \sum_{f=1}^{4}\left(\overline{\mathbf{F}}_{h h}^{i i, f} \mathbf{h}_{f}^{i}+\overline{\mathbf{F}}_{h h}^{i j, f} \mathbf{h}_{f}^{j}+\overline{\mathbf{F}}_{h e}^{i i, f} \mathbf{e}_{f}^{i}+\overline{\mathbf{F}}_{h e}^{i j, f} \mathbf{e}_{f}^{j}\right)
\end{aligned}
$$

where $\mathbf{M}_{\mathrm{e} / \mathrm{h}}^{i}$ and $\mathbf{S}_{e / h}^{i}$ are mass and stiffness matrices with dimension equal to number of the DoF in element $i, \overline{\mathbf{C}}_{\mathrm{WE}}^{i}$ is the thin wire-field coupling matrix defined in (35), $\overline{\mathbf{F}}_{e e}, \overline{\mathbf{F}}_{e h}$, $\overline{\mathbf{F}}_{h h}$, and $\overline{\mathbf{F}}_{h e}$ are matrices arising from the numerical flux. They are defined as follows:

$$
\begin{aligned}
& {\left[\overline{\mathbf{M}}_{e}^{i}\right]_{k l}=\int_{\Omega_{i}} \Phi_{k}^{i} \cdot \epsilon^{i} \Phi_{l}^{i} d V} \\
& {\left[\overline{\mathbf{M}}_{h}^{i}\right]_{k l}=\int_{\Omega_{i}} \Psi_{k}^{i} \cdot \mu^{i} \Psi_{l}^{i} d V} \\
& {\left[\overline{\mathbf{S}}_{e}^{i}\right]_{k l}=\int_{\Omega_{i}} \Phi_{k}^{i} \cdot \nabla \times \Psi_{l}^{i} d V} \\
& {\left[\overline{\mathbf{S}}_{h}^{i}\right]_{k l}=\int_{\Omega_{i}} \Psi_{k}^{i} \cdot \nabla \times \Phi_{l}^{i} d V} \\
& {\left[\overline{\mathbf{F}}_{e e}^{i i, f}\right]_{k l}=\frac{-1}{Z^{i}+Z_{f}^{j}} \int_{\partial \Omega_{i, f}} \Phi_{k}^{i} \cdot \hat{\mathbf{n}}_{i, f} \times\left(\hat{\mathbf{n}}_{i, f} \times \Phi_{l}^{i}\right) d S} \\
& {\left[\overline{\mathbf{F}}_{e e}^{i j, f}\right]_{k l}=\frac{1}{Z^{i}+Z_{f}^{j}} \int_{\partial \Omega_{i, f}} \Phi_{k}^{i} \cdot \hat{\mathbf{n}}_{i, f} \times\left(\hat{\mathbf{n}}_{i, f} \times \Phi_{l}^{j, f}\right) d S} \\
& {\left[\overline{\mathbf{F}}_{e h}^{i i, f}\right]_{k l}=-\frac{Z_{f}^{j}}{Z^{i}+Z_{f}^{j}} \int_{\partial \Omega_{i, f}} \Phi_{k}^{i} \cdot \hat{\mathbf{n}}_{i, f} \times \Psi_{l}^{i} d S}
\end{aligned}
$$




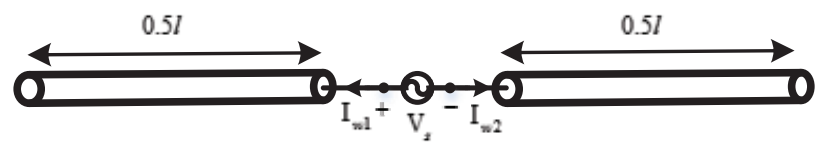

Fig. 5. A $l=15 \mathrm{~cm}$ dipole antenna is driven by a voltage source at its center.

TABLE I

THE NUMERICALLY OBTAINED

\begin{tabular}{c||cccc}
\multicolumn{4}{c}{ frequencies by the proposed algorithm. } \\
\hline \hline freq. [GHz] & 0.973 & 1.913 & 2.982 & 3.938 \\
\hline err. [\%] & 2.7 & 4.35 & 0.6 & 1.55 \\
\hline \hline
\end{tabular}

$$
\begin{gathered}
{\left[\overline{\mathbf{F}}_{e h}^{i j, f}\right]_{k l}=\frac{Z_{f}^{j}}{Z^{i}+Z_{f}^{j}} \int_{\partial \Omega_{i, f}} \Phi_{k}^{i} \cdot \hat{\mathbf{n}}_{i, f} \times \Psi_{l}^{j, f} d S} \\
{\left[\overline{\mathbf{F}}_{h h}^{i i, f}\right]_{k l}=\frac{-1}{Y^{i}+Y_{f}^{j}} \int_{\partial \Omega_{i, f}} \Psi_{k}^{i} \cdot \hat{\mathbf{n}}_{i, f} \times\left(\hat{\mathbf{n}}_{i, f} \times \Psi_{l}^{i}\right) d S} \\
{\left[\overline{\mathbf{F}}_{h h}^{i j, f}\right]_{k l}=\frac{1}{Y^{i}+Y_{f}^{j}} \int_{\partial \Omega_{i, f}} \Psi_{k}^{i} \cdot \hat{\mathbf{n}}_{i, f} \times\left(\hat{\mathbf{n}}_{i, f} \times \Psi_{l}^{j, f}\right) d S} \\
{\left[\overline{\mathbf{F}}_{h e}^{i i, f}\right]_{k l}=\frac{Y_{f}^{j}}{Y^{i}+Y_{f}^{j}} \int_{\partial \Omega_{i, f}} \Psi_{k}^{i} \cdot \hat{\mathbf{n}}_{i, f} \times \Phi_{l}^{i} d S} \\
{\left[\overline{\mathbf{F}}_{h e}^{i j, f}\right]_{k l}=\frac{-Y_{f}^{j}}{Y^{i}+Y_{f}^{j}} \int_{\partial \Omega_{i, f}} \Psi_{k}^{i} \cdot \hat{\mathbf{n}}_{i, f} \times \Phi_{l}^{j, f} d S}
\end{gathered}
$$

The semi-discrete matrix equations in (40) and (41) are solved by the explicit fourth-order RK method. To ensure stability, the time step size is determined according to the following condition:

$$
c_{0} \delta t_{D G} \leq \min \left\{l_{\min } \sqrt{\epsilon_{r} \mu_{r}} / 4(p+1)^{2}\right\}
$$

where $c_{0}$ is the free-space light speed, $p$ is equal to one in this work.

Since the time step sizes for the thin wire and the Maxwell's equations solvers are different, the minimum one is used for simplicity and uniform in this work, i.e., $\delta t=$ $\min \left\{\delta t_{w}, \delta t_{D G}\right\}$.

\section{NUMERICAL RESULTS}

In this part, three representative examples are provided to verify the accuracy of the proposed algorithm. According to the previous literatures, the radius $\rho_{0}$ of the coupling tube zone plays a critical role in the determination of accuracy of the wire-field coupling. Based on the previous numerical experiments [4], [5], $\rho_{0}$ in this work is set to be $1.7 \Delta$ with $\Delta$ denoting the average edge length of the mesh cells surrounding the wire.

\section{A. A Dipole Antenna}

In the first example, a $l=15 \mathrm{~cm}$ dipole antenna $(a=0.25$ $\mathrm{mm}$ ) driven by a voltage source at its central point is characterized, as shown in Fig. 5. We split it into 20 wire segments. The dimension of the wire-circuit coupling matrix is 5 by 5 , including two auxiliary current sources $I_{w 1}$ and $I_{w 2}$, two nonreference nodal voltages, and one current flowing through the voltage source branch. For this open-space radiation problem, the computational domain must be truncated by artificial boundaries for partial differential equation (PDE) solvers. In this work, the hybrid DGTD and time-domain boundary integral (TDBI) algorithm [35] is applied. At the truncation boundary, the field values $\left(\mathbf{E}_{\partial \Omega}^{j}, \mathbf{H}_{\partial \Omega}^{j}\right)$ at the outside of the computational domain are not available to evaluate the incoming numerical flux. To handle this issue, the TDBI is employed to calculate these field values based on the Huygens' principle. The hybrid DGTD-BI method is mathematically rigorous and the resultant truncation boundary can be conformal to arbitrary scatterers. More importantly, disconnected scatterers can be locally truncated by their own conformal boundary instead of using a global truncation boundary in FEM-BI [36], [37]. Compared with the time step size of DGTD, TDBI have much more larger one. In this work, temporal interpolation is conducted [35]. Totally 12,755 tetrahedrons are involved, and the corresponding time step sizes of DGTD and TDBI are $0.4985 \mathrm{ps}$ and $7.886 \mathrm{ps}$, respectively. The average edge length $\Delta$ in the proximity of the dipole is $0.76 \mathrm{~cm}$. To verify the accuracy of the proposed algorithm, the frequencies for $l=\lambda / 2, l=\lambda, l=3 \lambda / 2$, and $l=2 \lambda$ are calculated by the proposed algorithm, as shown in Table I. It is interestingly noted that all numerically obtained frequencies are little smaller than the analytical results, which are attributed to that the end-cap effects of finite length thin wires are not included in the thin wire model (The accumulation of the charge at the end of the thin wire will alter the current distribution along the wire) [38]. The normalized far-field radiation patterns for the first three frequencies are shown in Fig. 6. For comparison, we also provide the analytical results calculated by [39]

$$
F_{\theta}=\frac{\cos (k l \cos (\theta) / 2)-\cos (k l / 2)}{\sin (\theta)},
$$

where $k$ and $\theta$ denote the wave number and the angle between $\mathbf{R}$ and $z$-axis, respectively.

\section{B. PEC Shielding Enclosure with a Probe Feeding}

As the second example, a PEC shielding enclosure [24] is fed by a $14 \mathrm{~cm}$ coaxial probe with radius $a=0.8 \mathrm{~mm}$, as shown in Fig. 7. At the near-end, the probe is driven by a voltage source defined as

$$
\begin{aligned}
V_{s}= & e^{-\alpha_{1}^{2}\left(f_{2}-f_{1}\right)^{2}\left(t-\frac{\alpha_{2}}{f_{2}-f_{1}}\right)^{2}} \\
& \times \cos \left[2 \pi \frac{f_{1}+f_{2}}{2}\left(t-\frac{\alpha_{2}}{f_{2}-f_{1}}\right)\right]
\end{aligned}
$$

with $f_{1}=0.7 \mathrm{GHz}, f_{2}=1.6 \mathrm{GHz}, \alpha_{1}=1.035$ and $\alpha_{2}=2.539$. At the far-end, the probe is terminated by a $47 \Omega$ resistor.

To facilitate the thin wire solver, we uniformly split the 


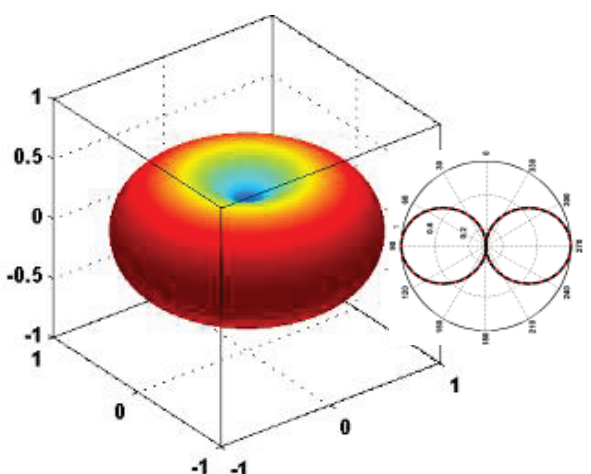

(a)

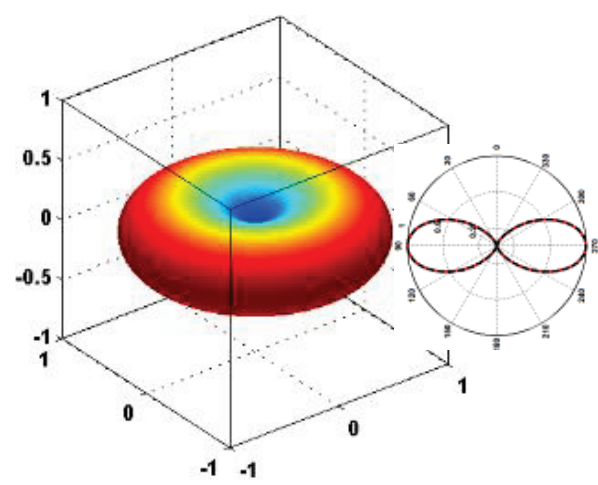

(b)

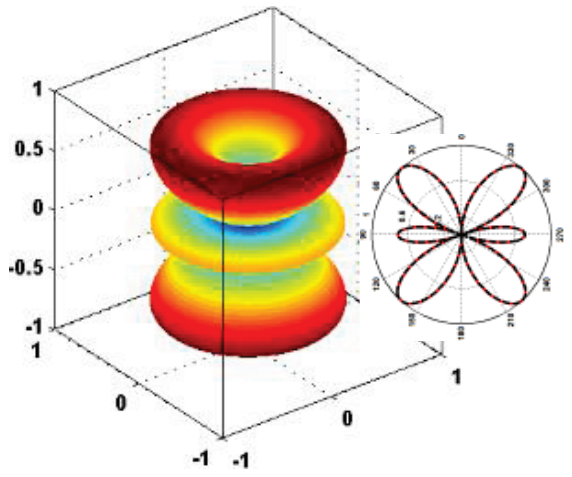

(c)

Fig. 6. The calculated far-field patterns at $f_{1}=0.9726 \mathrm{GHz}(\mathrm{a}), f_{2}=1.913 \mathrm{GHz}$ (b), and $f_{3}=2.982 \mathrm{GHz}$ (c). For comparison, the analytical results denoted by the dashed black line are also provided.

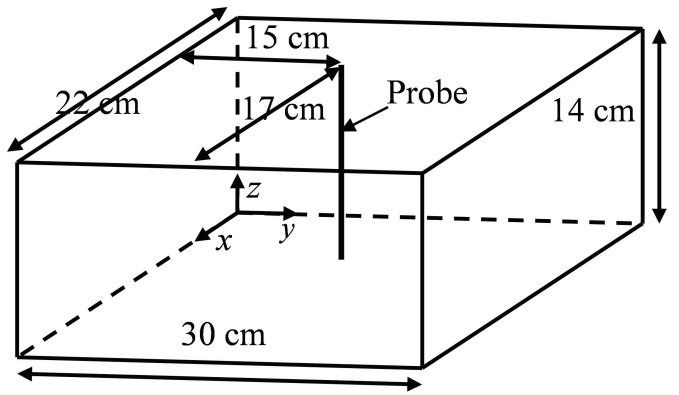

(a)

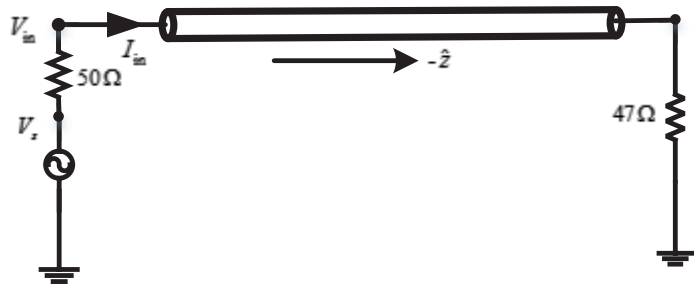

(b)

Fig. 7. The schematic illustration of the shielding enclosure fed with a probe [24] (a) and the details of probe-circuit interface (b).

probe into 14 segments. The shielding cube is discretized into 22,275 tetrahedrons, and the average edge length $\Delta$ in the vicinity of the thin wire is around $1.56 \mathrm{~cm}$. The corresponding time step size $\delta t=1.0331 \mathrm{ps}$. To demonstrate the accuracy of the proposed algorithm, the power delivered to the probe is calculated and compared with the measurement result. Since the measurement reference is conducted in the frequency domain with $1 \mathrm{~V}$ input voltage, the obtained frequency domain voltage and current via fast Fourier transform (FFT) are thus normalized to the corresponding input voltage after FFT [5]. Namely,

$$
P_{d}(\omega)=\frac{1}{2} \mathfrak{R e}\left\{\frac{V_{\mathrm{in}}(\omega)}{V_{\mathrm{s}}(\omega)} \times \frac{I_{\mathrm{in}}^{*}(\omega)}{V_{\mathrm{s}}^{*}(\omega)}\right\}
$$

where the superscript $*$ denotes the complex conjugate.

In Fig. 8 , the recorded time-domain voltage $V_{\text {in }}(t)$ and current $I_{\text {in }}(t)$ are shown. Due to the strong cavity resonance,

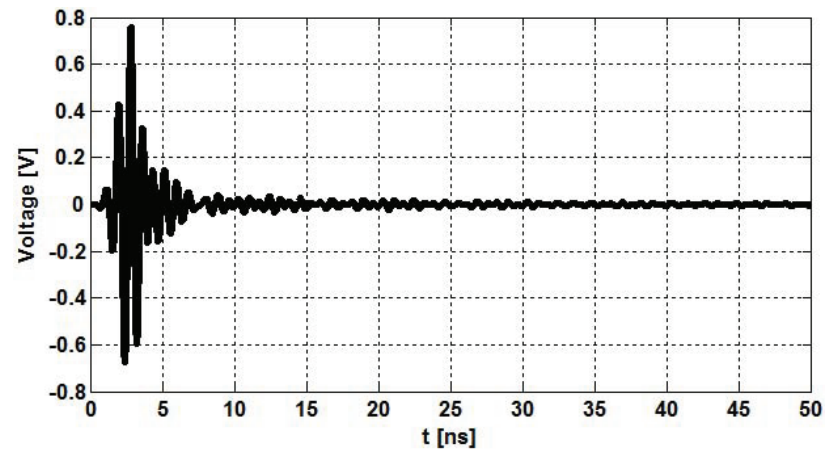

(a)

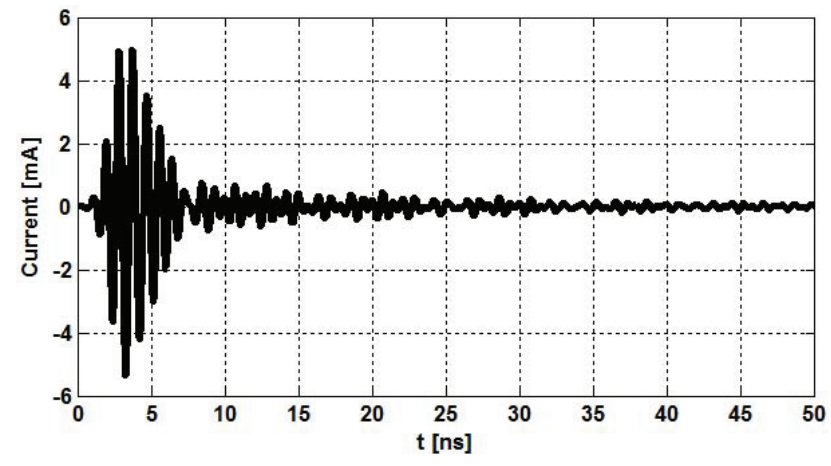

(b)

Fig. 8. The voltage and current delivered into the probe are plotted versus time to $50 \mathrm{~ns}$.

a long tail is observed. The corresponding power delivered to the probe is calculated according to (46) and shown in Fig. 9. For comparison, the measurement reference [24] is also given. It is noted that very good agreements are achieved.

Next, a more complex situation is considered by placing a microwave metal-semiconductor-field-effect transistor (MESFET) amplifier [16], [30] as shown in Fig. 10 between the $50 \Omega$ resistor and the near-end of the probe. Due to the introduction of capacitors and inductors in the equivalent circuit model of MESFET, the resonant frequencies of the probe might be alternated. We let the transconductance $G_{m}$ that determines the gain of the MESFET as a parameter, and then calculate the power delivered to the probe for 
TABLE II

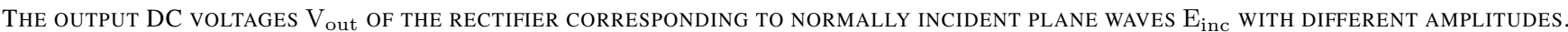

\begin{tabular}{|c|c|c|c|c|c|c|c|c|c|c|c|c|c|c|c|c|}
\hline $\mathrm{E}_{\text {inc }}[\mathrm{V} / \mathrm{m}]$ & 0.25 & 0.5 & 0.75 & 1.0 & 1.25 & 1.5 & 1.75 & 2.0 & 2.25 & 2.5 & 2.75 & 3.0 & 3.25 & 3.5 & 3.75 & 4.0 \\
\hline $\mathrm{V}_{\text {out }}[\mathrm{V}]$ & 0.19 & 0.54 & 0.98 & 1.44 & 1.92 & 2.41 & 2.91 & 3.41 & 3.92 & 4.43 & 4.94 & 5.45 & 5.96 & 6.48 & 6.99 & 7.51 \\
\hline
\end{tabular}

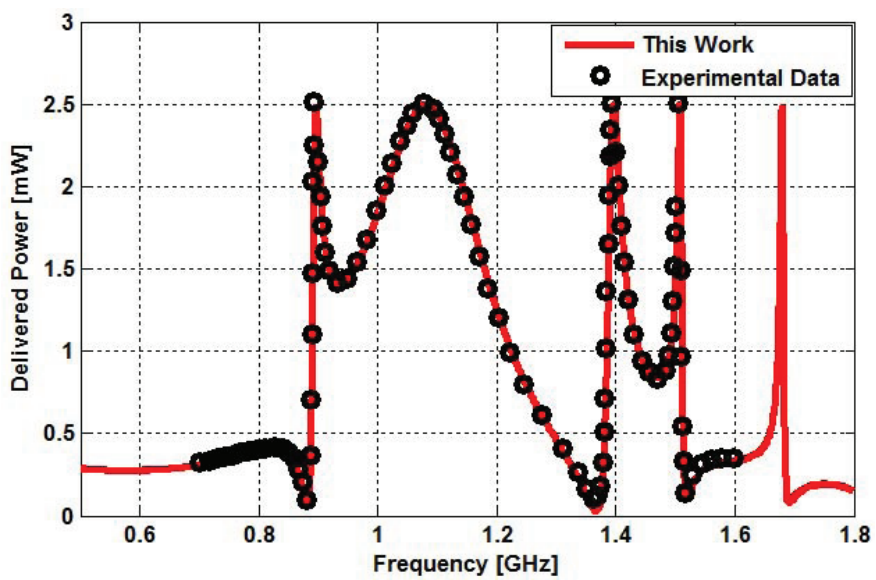

Fig. 9. The calculated power delivered to the probe by the proposed algorithm. For comparison, the measurement result is also provided.

different $G_{m}$. In Fig. 11, the delivered powers corresponding to $G_{m}=25,35,42.5$, and $50 \mathrm{mS}$ are presented. As expected, the original resonant frequency of probe is changed from 1.08 $\mathrm{GHz}$ to $1.165 \mathrm{GHz}$. Simultaneously, the magnitudes of the resonant peaks are also varied. All these can be attributed to the frequency dependent gain of the MESFET amplifier and the impedance matching condition.

\section{A Monopole Rectenna}

For the third example, a monopole antenna loaded with a rectifier is studied. As shown in Fig. 12, the $15 \mathrm{~cm}$ monopole antenna is place along the $z$-direction with $a=1 \mathrm{~mm}$, and a $z$-directed sinusoidally modulated plane wave propagating along the $y$-direction is employed as the excitation. The rectifier comprises of a three-stage differential mode voltage multiplier [33]. Each diode in this work is described by a standard exponential current source $I_{d}=I_{0}\left[e^{V_{d} / V_{0}}-1\right]$ with $I_{0}=1.0 \mu \mathrm{A}$ and $V_{0}=0.03 \mathrm{~V}$. The values of reactive elements are given as: $L_{0}=54.3 \mathrm{nH}, C_{0}=0.55 \mathrm{pF}, C_{1}=1 \mathrm{pF}$, and $C_{2}=1 \mathrm{pF}$. The total field/scattered field (TF/SF) technique is utilized to implement the plane wave excitation in this work. To implement the TF/SF into DGTD, the numerical flux at the TF/SF interface should be corrected as follows: in the $\mathrm{TF}$ region, the incoming flux from the $\mathrm{SF}$ region is modified as: $\mathbf{E}^{j}=\mathbf{E}_{\mathrm{SF}}^{j}+\mathbf{E}_{\text {inc }}$ and $\mathbf{H}^{j}=\mathbf{H}_{\mathrm{SF}}^{j}+\mathbf{H}_{\text {inc }}$; in the $\mathrm{SF}$ region, the field values for incoming flux calculation are revised as: $\mathbf{E}^{j}=\mathbf{E}_{\mathrm{TF}}^{j}-\mathbf{E}_{\mathrm{inc}}$ and $\mathbf{H}^{j}=\mathbf{H}_{\mathrm{TF}}^{j}-\mathbf{H}_{\mathrm{inc}}$.

For this example, the monopole is uniformly meshed into 10 segments, and the field domain is split into 4,908 tetrahedrons. The resulted time-step sizes of DGTD and TDBI are 1.085 ps and $15.77 \mathrm{ps}$, respectively. The average edge length in

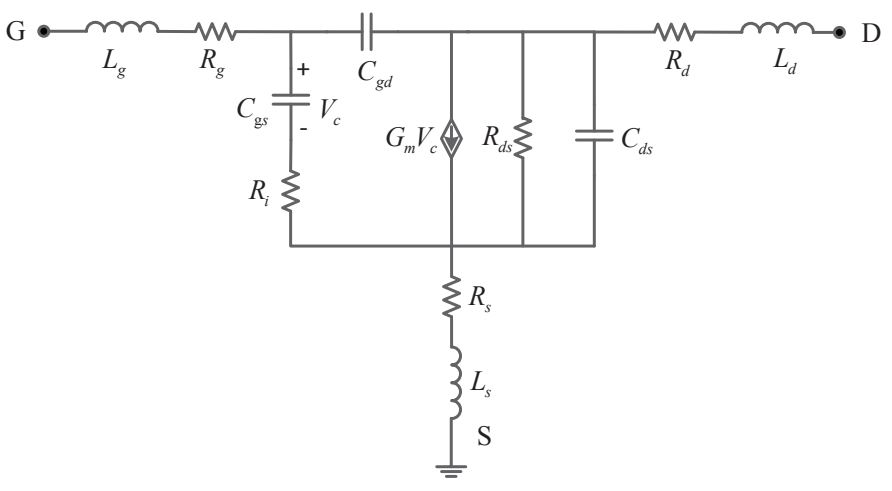

Fig. 10. The extrinsic equivalent circuit model of a small signal MESFET. $L_{g}=0.37 \mathrm{nH}, L_{d}=0.23 \mathrm{nH}, L_{s}=0.02 \mathrm{nH}, C_{g d}=0.06 \mathrm{pF}, C_{d s}=$ $0.26 \mathrm{pF}, C_{g s}=0.69 \mathrm{pF}, R_{g}=1.39 \Omega, R_{s}=0.76 \Omega, R_{i}=1.42 \Omega$, $R_{d s}=197 \Omega, R_{d}=1.3 \Omega$, and the transconductance $G_{m}$ is a parameter to be determined.

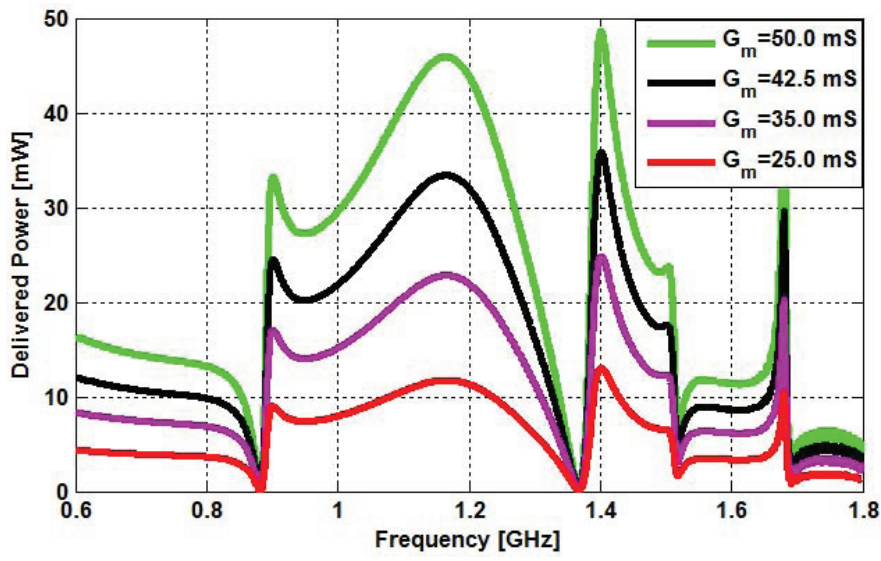

Fig. 11. The power delivered to the thin wire via a MESFET amplifier with varying transconductance.

the proximity of the monopole is around $1.53 \mathrm{~cm}$. The number of unknowns of the resultant wire-circuit matrix is 15 , including fourteen nodal voltage and one auxiliary current source unknowns. Due to the nonlinear property of the diode, the Newton-Raphson method is employed with the iteration number equal to 15 . Firstly, the output DC voltage versus plane waves with different amplitudes are investigated, as shown in Table II. In Fig. 13, the recorded output voltage $\mathrm{V}_{\text {out }}$ versus the time $t$ is plotted for incident wave with amplitude $\mathrm{E}_{\mathrm{inc}}=3.0 \mathrm{~V} / \mathrm{m}$. As expected, the $\mathrm{AC}$ input is successfully converted to a DC output with amplitude around $5.448 \mathrm{~V}$. For realistic situations, the incident waves could impinge on the antenna with different directions of arrival. Thus, its is necessary to study the output DC voltage for 


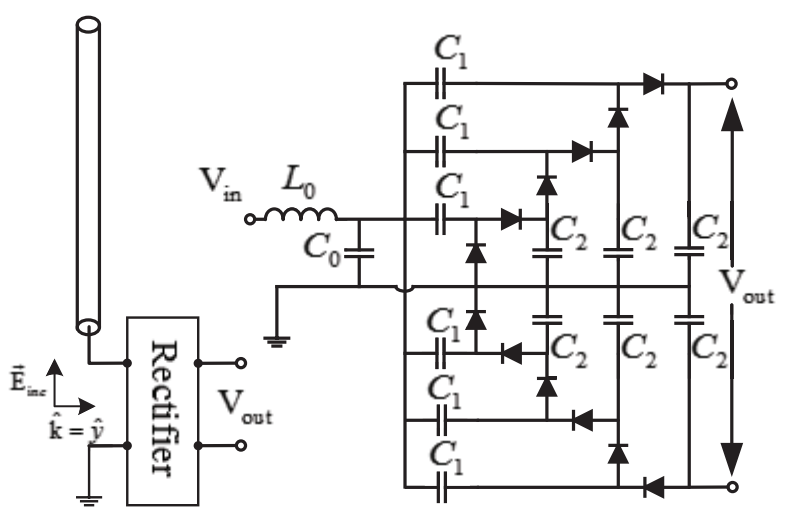

Fig. 12. Left: A monopole rectenna under the illumination of a normally incident plane wave. Right: The circuit details of the rectifier composed of a three-stage voltage multiplier.

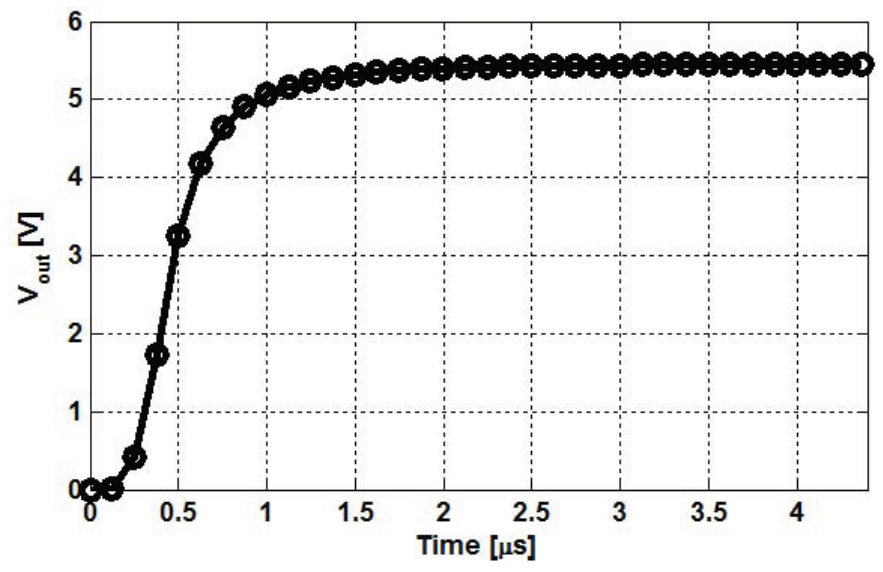

Fig. 13. The temporal evolution of the voltage at the output port corresponding to a normally incident wave with amplitude $\mathrm{E}_{\text {inc }}=3.0 \mathrm{~V} / \mathrm{m}$.

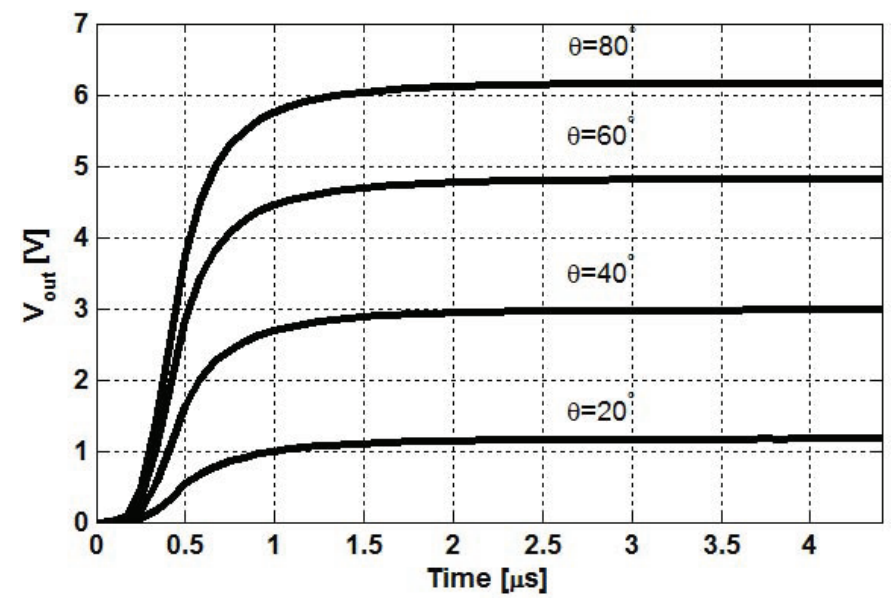

Fig. 14. The output voltage $\mathrm{V}_{\text {out }}$ corresponding to the different obliquely incident wave with amplitude $\mathrm{E}_{\mathrm{inc}}=3.5 \mathrm{~V} / \mathrm{m} . \theta$ denotes the angle between the wave number $\hat{\mathbf{k}}$ and the positive $z$-axis.

obliquely incident waves. In Fig. 14, the output voltages for plane waves propagating in the $y z$ plane with wave number $\hat{\mathbf{k}}=\sin (\theta) \hat{\mathbf{y}}+\cos (\theta) \hat{\mathbf{z}}$ are plotted as the function of time. It is noted that the output voltage decreases with the incident angle, which agrees with the theory.

Next, to investigate the impacts of the number of voltage

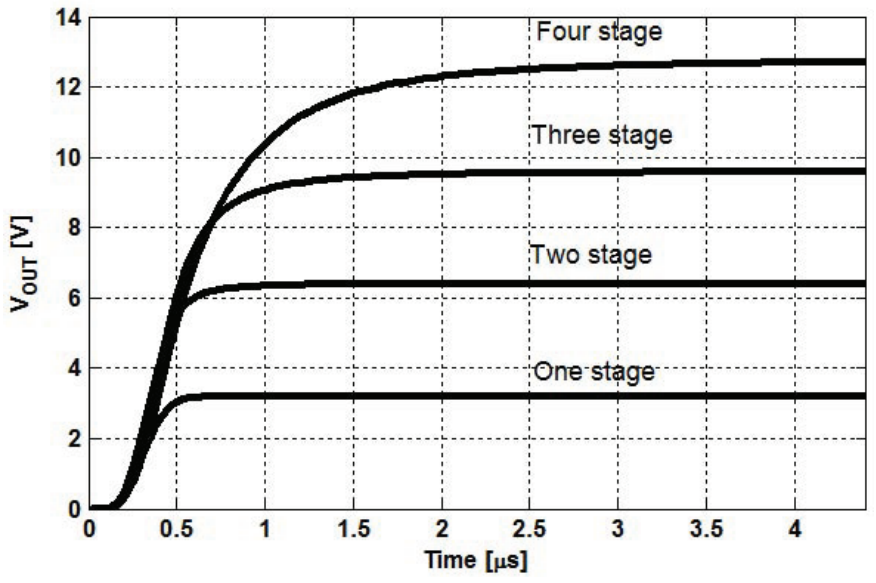

Fig. 15. The output DC voltages corresponding to rectifiers with one to four-stage voltage multipliers.

multipliers on the magnitude of output voltage, a normally incident plane wave with amplitude $\mathrm{E}_{\mathrm{in}}=5 \mathrm{~V} / \mathrm{m}$ is employed as the excitation. In Fig. 15, the recorded temporal results with one to four voltage multipliers are plotted. The numbers of wire-circuit coupling matrix unknowns are $6,11,15$, and 19, respectively. As expected, the amplitude of the output voltage increases with the number of voltage multipliers. Interestingly, it is noted that it takes more time to reach steady state for the case with more voltage multipliers.

\section{CONCLUSION}

In this paper, a thin wire model characterized by a modified telegrapher's equation (MTE) is employed to replace the physical presence of the thin wire, which results in a reduced number of unknowns and relaxed CFL number. To treat thin wires loaded by lumped circuits, auxiliary equations derived from the invariable property of the characteristic variables are integrated into the MNA based circuit equations. The thin wire-circuit coupling matrix equation is iteratively solved by Newton-Raphson method if nonlinear circuit elements are involved. As hyperbolic systems, both the MTEs and the Maxwell's equations are solved by the DGTD with explicit time-marching scheme. For the open-region problems, the hybrid DGTD-BI approach is used to conformal and locally truncate the computational domain. The accuracy of the proposed algorithm is demonstrated by three representative examples.

\section{REFERENCES}

[1] J. Shi, M. A. Cracraft, K. P. Slattery, M. Yamaguchi, and R. E. Dubroff, "Calibration and compensation of near-field scan measurements," IEEE Trans. Electromagn. Compat., vol. 47, no. 3, pp. 642-650, Aug. 2005.

[2] H. Zhao and Z. Shen, "Weighted Laguerre polynomials-finite difference method for time-domain modeling of thin wire antennas in a loaded cavity," IEEE Antennas Wireless Propag. Lett., vol. 8, pp. 1131-1134, 2008.

[3] R. Holland and L. Simpson, "Finite-difference analysis of EMP coupling to thin struts and wires," IEEE Trans. Electromagn. Compat., vol. 23, no. 2, pp. 88-97, May 1981 .

[4] F. Edelvik, "A new technique for accurate and stable modeling of arbitrarily oriented thin wires in the FDTD method," IEEE Trans. Electromagn. Compat., vol. 45, no. 2, pp. 416-423, May 2003. 
[5] F. Edelvik, G. Ledfelt, P. Lotstedt, and D. J. Riley, "An unconditionally stable subcell model for arbitrarily oriented thin wires in the FETD method," IEEE Trans. Antennas Propag., vol. 51, no. 8, pp. 1797-1805, Aug. 2003.

[6] T. Noda and S. Yokoyama, "Thin wire representation in finite difference time domain surge simulation," IEEE Trans. Power Del., vol. 17. no. 3, pp. 840-847, Jul. 2002.

[7] Y. Taniguchi, Y. Baba, N. Nagaoka, and A. Ametani, "An improved thin wire representation for FDTD computations," IEEE Trans. Antennas Propag., vol. 56, no. 10, pp. 3248-3252, Oct. 2008.

[8] S. H. Lee and J. M. Jin, "Fast reduced-order finite-element modeling of lossy thin wires using lumped impedance elements," IEEE Trans. Adv. Packag., vol. 33, no. 1, pp. 212-218, Feb. 2010.

[9] T. Asada, Y. Baba, N. Nagaoka, and A. Ametani, "An improved thin wire representation for FDTD transient simulations," IEEE Trans. Electromagn. Compat., in Press.

[10] A. G. Tijhuis, Z. Peng, and A. R. Bretones, "Transient excitation of a straight thin-wire segment: a new look at an old problem," IEEE Trans. Antennas Propag., vol. 40, no. 10, pp. 1132-1146, Oct. 1992.

[11] B. Rynne, "On the well-posedness of Pocklington's equation for a straight wire antenna and convergence of numerical solutions," J. Electromagn. Waves Applicat., vol. 14, pp. 1489-1503, 2000.

[12] E. Forati, A. D. Mueller, P. G. Yarandi, and G. W. Hanson, "A new formulation of Pocklington's equation for thin wires using the exact kernel," IEEE Trans. Antennas Propag., vol. 59, no. 11, pp. 4355-4360, Nov. 2011.

[13] C. Christopoulos, An Introduction to the Transmission-Line Modeling TLM Method. New York: IEEE Press, 1992.

[14] P. Li and L. J. Jiang, "Simulation of electromagnetic waves in the magnetized cold plasma by a DGFETD method," IEEE Antennas Wireless Propag. Lett., vol. 12, pp. 1244-1247, 2013.

[15] J. S. Hesthaven and T. Warburton, Nodal Discontinuous Galerkin Methods. Berlin: Springer, 2008.

[16] P. Li and L. J. Jiang, "Integration of arbitrary lumped multiport circuit networks into discontinuous Galerkin time-domain analysis," IEEE Trans. Microw. Theory and Techn., vol. 61, no. 7, pp. 2525-2534, Jul. 2013.

[17] P. Li, Y. Shi, L. J. Jiang, and H. Bagci, "A DGTD scheme for modeling the radiated emission from DUTs in sheilding enclosures using near electric field only," IEEE Trans. Electromagn. Compat., vol. pp. no. 99, pp. 1-11, Jan. 2016.

[18] R. J. Leveque, Finite Volume Methods for Hyperbolic Problems. Cambridge, U.K.: Cambridge Univ. Press, 2002.

[19] K. Sankaran, "Accurate domian truncation techniques for time-domain conformal methods," Ph.D. dissertation, Dept. Inform. Technol. Elect. Eng., ETH Zurich, Zurich, Switzerland, 2007.

[20] I. Jeffrey and J. Lovetri, "Interfacing thin-wire and circuit subcell models in unstructured time-domain field solvers," IEEE Trans. Antennas Propag., vol. 60, no. 4, pp. 1978-1986, Apr. 2012.

[21] J. M. Jin, The Finite Element Method in Electromagnetics, 2nd ed. New York, NY, USA: Wiley, 2003.

[22] X. Li and J. M. Jin, "A comparative study of three finite elementbased explicit numerical schemes for solving Maxwell's equations," IEEE Trans. Antennas Propag., vol. 60, no. 3, pp. 1450-1457, Mar. 2012.

[23] M. Bingle, D. B. Davidson, and J. H. Cloete, "Scattering and absorption by thin metal wires in rectangular waveguide-FDTD simulation and physical experiments," IEEE Trans. Microw. Theory Tech., vol. 50, n0. 6, pp. 1621-1627, Jun. 2002.

[24] M. Li, K. P. Ma, D. M. Hockanson, J. L. Drewniak, T. H. Hubing, and T. P. V. Doren, "Numerical and experimental corroboration of an FDTD thin-slot model for slots near corners of shielding enclosures," IEEE Trans. Electromagn. Compat., vol. 39, no. 3, pp. 225-232, Aug. 1997.

[25] S. Hwang, S. Burintramart, T. K. Sarkar, and S. R. Best, "Direction of arrival estimation using electrically small tuned dipole antennas," IEEE Trans. Antennas Propag., vol. 54, no. 11, pp. 3292-3301, Nov. 2006.

[26] B. R. Jackson, S. Rajan, B. Liao, and S. Wang, "Direction of arrival estimation using directive antennas in uniform circular arrays," IEEE Trans. Antennas Propag., early access, 2014.

[27] C. Ho, A. E. Ruehli, and P. A. Brennan, "The modified nodal approach to newtork analysis," IEEE Trans. Circuits Syst., vol. CAS-22, no. 6, pp. 504-509, Jun. 1975.

[28] P. Li, L. J. Jiang, and H. Bagci, "Cosimulation of electromagneticscircuit systems exploiting DGTD and MNA," IEEE Trans. Components, Packaging, and Manufacturing Technology, vol. 4, no. 6, pp. 1052-1061, Jun. 2014
[29] P. Li and L. J. Jiang, "A hybrid electromagnetics-circuit simulation method exploiting discontinuous Galerkin finite element time domain method," IEEE Microw. Wireless Compon. Lett., vol. 23, no. 3, pp. 113115, Mar. 2013.

[30] C. C. Wang and C. W. Kuo, "An efficient scheme for processing arbitrary lumped multiport devices in the finite-difference time-domain method," IEEE Trans. Microw. Theory Tech., vol. 55, no. 5, May 2007.

[31] D. B. Davidson, "An evaluation of mixed-order versus full-order vector finite elements," IEEE Trans. Antennas Propag., vol. 51, no. 9, pp. 24302441, Sept. 2003.

[32] J. P. Webb, "Hierarchal vector basis functions of arbitrary order for triangular and tetrahedral finite elements," IEEE Trans. Antennas Propag., vol. 47 , no. 8, pp. 1244-1253, Aug. 1999.

[33] J. Curty, N. Joehl, F. Krummenacher, C. Dehollain, and M. J. Declerq, "A model for $\mu$-power rectifier analysis and design," IEEE Trans. Circuits Syst. I, Reg. Papers, vol. 52, no. 12, pp. 2771-2779, Dec. 2005.

[34] P. Li, Y. Shi, L. J. Jiang, and H. Bağc1, "A hybrid time-domain discontinuous galerkin-boundary integral method for electromagnetic scattering analysis," IEEE Trans. Antennas Propag., vol. 62, no. 5, pp. 2841-2846, May 2014.

[35] P. Li, Y. Shi, L. J. Jiang, and H. Bağc1, "A hybrid time-domain discontinuous galerkin-boundary integral method for electromagnetic scattering analysis," IEEE Trans. Antennas Propag., vol. 62, no. 5, pp. 2841-2846, May 2014.

[36] D. Jiao, M. Lu, E. Michielssen, and J. M. Jin, "A fast time-domain finite element-boundary integral method for electromagnetic analysis," IEEE Trans. Antennas Propag., vol. 49, no. 10, pp. 1453-1461, Oct. 2001.

[37] D. Jiao, A. A. Ergin, B. Shanker, E. Michielssen, and J. M. Jin, "A fast higher-order time-domain finite element-boundary integral method for 3D electromagnetic scattering analysis," IEEE Trans. Antennas Propag., vol. 50, no. 9, pp. 1192-1202, Sept. 2002.

[38] J. A. Huffman and D. H. Werner, "Modeling of a cylindrical wire antenna with fiat end caps using a rigorous moment method technique, "Radio Science, vol. 36, no. 3, pp. 407-425, May 2001.

[39] C. A. Balanis, Antenna Theory Analysis and Design, Third Edition, John Wiley \& Sons, 2005.

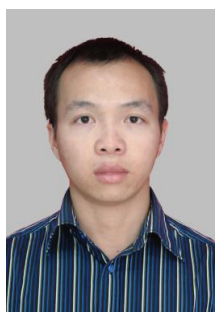

Ping Li (S'12-M'15) has been working toward the $\mathrm{Ph} . \mathrm{D}$. degree with the center of Electromagnetics and Optics at the University of Hong Kong since 2010. $\mathrm{He}$ is the recipient of the Postgraduate Engineering Fellowship from 2010. He is listed in Marquis Who's Who in the World, $32^{\text {nd }}$ Edition, 2015.

He has authored over 10 journal papers on IEEE Trans. Microwave Theory and Techniques, IEEE Trans. Antennas and Propagation, IEEE Trans. Electromagnetic and Compatibility, IEEE Trans. Components, Packaging, and Manufacturing Technologies, etc. His paper was selected as the Finalist paper in 29-th International Review of Progress in Applied Computational Electromagnetics and 2014 International Symposium on Electromagnetic Compatibility, and he won the best student paper award in 12-th International Workshop on Finite Elements for Microwave Engineering. He served as the reviewer of IEEE Trans. Microwave Theory and Techniques, IEEE Trans. Antennas and Propagation, IEEE Antennas and Wireless Propagation Letters, Proceedings of IEEE, Progress in Electromagnetics Research Letters, Journal of Electromagnetic Waves and Applications, Journal of ACES

His research interests include the near-field to far-field transformation techniques, phaseless equivalent source reconstruction methods, discontinuous Galerkin time-domain method, and uncertainty quantification for large scale electromagnetic systems. 


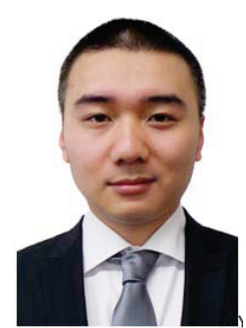

Yifei Shi (S'09-M'12) was born in Jiangsu, China, in 1981. He received the B.E. degree in electronic and information engineering from Nanjing Tech University, Nanjing, Jiangsu, China, in 2004. He received the Ph.D. degree in electromagnetic field and microwave technology from Nanjing University of Science and Technology, Nanjing, Jiangsu, China, in 2012. Since 2012 he is a Postdoctoral Research Fellow in the Department of Electrical Engineering, King Abdullah University of Science and Technology, Thuwal, Saudi Arabia. His research interest is time domain integral equation.

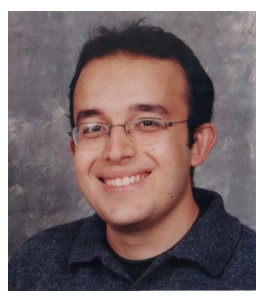

Hakan Bağcii (S'98-M'07-S'14) received the B.S. degree in Electrical and Electronics Engineering from the Bilkent University, Ankara, Turkey, in June 2001 and the M.S. and Ph.D. degrees in Electrical and Computer Engineering from the University of Illinois at Urbana-Champaign (UIUC), Urbana, in August 2003 and January 2007, respectively. From June 1999 to July 2001, he worked as an Undergraduate Researcher at the Computational Electromagnetics Group, Bilkent University. From August 2001 to December 2006, he was a Research Assistant at the Center for Computational Electromagnetics and Electromagnetics Laboratory, UIUC. From January 2007 to August 2009, he worked as a Research Fellow at the Radiation Laboratory, University of Michigan. In August 2009, he joined the Division of Physical Sciences and Engineering at the King Abdullah University of Science and Technology (KAUST) as Assistant Professor of Electrical Engineering. His research interests include various aspects of computational electromagnetics with emphasis on time-domain integral equations and their fast marching-on-in-time-based solutions, wellconditioned integral-equation formulations, and development of fast hybrid methods for analyzing statistical EMC/EMI phenomena on complex and fully loaded platforms.

Dr. Bağc1 was the recipient of the 2008 International Union of Radio Scientists (URSI) Young Scientist Award and the 2004-2005 Interdisciplinary Graduate Fellowship from the Computational Science and Engineering Department, UIUC. His paper titled "Fast and rigorous analysis of EMC/EMI phenomena on electrically large and complex structures loaded with coaxial cables" was one of the three finalists (with honorable mention) for the 2008 Richard B. Schulz Best Transactions Paper Award given by the IEEE Electromagnetic Compatibility Society. He authored and co-authored eight finalist papers in the student paper competitions at the 2005, 2008, and 2010 IEEE Antennas and Propagation Society International Symposiums and 2013 and 2014 Applied Computational Electromagnetics Society Conference.

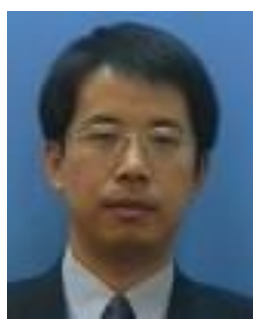

Li Jun Jiang (S'01-M'04-S'13) received the B.S. degree in Electrical Engineering from the Beijing University of Aeronautics and Astronautics in 1993, the M.S. degree in Electronic Engineering from the Tsinghua University in 1996, and Ph.D. in Electrical and Computer Engineering from the University of Illinois at Urbana-Champaign in 2004. From 1996 to 1999 , he was an application engineer with the Hewlett-Packard Company. Since 2004, he has been the postdoctoral researcher, the research staff member, and the senior engineer at IBM T.J. Watson Research Center. Since the end of 2009, he is an Associate Professor with the Department of Electrical and Electronic Engineering at the University of Hong Kong.

He received the IEEE MTT Graduate Fellowship Award in 2003 and the Y.T. Lo Outstanding Research Award in 2004. He is an IEEE Senior Member, the Associate Editor of IEEE Transactions on Antennas and Propagation, the Associate Editor of Progress in Electromagnetics Research, the Associate Guest Editor of the Proceedings of IEEE Special Issue in 2011 2012, an IEEE AP-S Member, an IEEE MTT-S member, an IEEE EMC-S member, an ACES member, and a member of Chinese Computational Electromagnetics Society. He was the Semiconductor Research Cooperation (SRC) Industrial Liaison for several academic projects. He served as the Scientific Consultant to Hong Kong ASTRI (Hong Kong Applied Science and Technology Research Institute Company Limited) in 2010-2011, the Panelist of the Expert Review Panel (ERP) of Hong Kong R\&D Centre for Logistics and Supply Chain Management Enabling Technologies since Jan. 1st, 2013. He is also the senior visiting professor at Tsinghua University since Jun. 2013.

$\mathrm{He}$ was the TPC Chair of the 7th International Conference on Nanophotonics (ICNP)/the 3rd Conference on Advances in Optoelectronics and Micro/Nano Optics (AOM), the TPC Co-chair of the 12th International Workshop on Finite Elements for Microwave Engineering, the Co-chair of 2013 International Workshop on Pulsed Electromagnetic Field at Delft, the Netherlands, the General Chair of 2014 IEEE 14th HK AP/MTT Postgraduate Conference. He was the elected TPC member of IEEE EPEP since 2014 the TPC member of IEEE EDAPS since 2010, the TPC member of 2013 IEEE ICMTCE, the scientific committee member of 2010 IEEE SMEE, the special session organizers of IEEE EDAPS, IEEE EMC, ACES, APRASC, PIERS, co-organizer of HKU Computational Science and Engineering Workshops in 2010-2012, the TC-9 and TC-10 member of IEEE EMC-S since 2011, and session chairs of many international conferences. He also serves as the reviewer of IEEE Transactions on several topics, and other primary electromagnetics and microwave related journals.

.

